Nota Científica

\title{
Influência do comprimento de copa na relação hipsométrica de Araucaria angustifolia
}

Sebastião do Amaral Machado', Yuri Accioly¹, Rodrigo Geroni Mendes Nascimento', Luís César Rodrigues da Silva', Claudia Carla Cardozo ${ }^{1}$

${ }^{1}$ Universidade Federal do Paraná, Avenida Prefeito Lothário Meissner, 900, CEP 80210-170, Jardim Botânico, Curitiba, PR, Brasil.

"Autor correspondente:

samachado@ufpr.br

Termos para indexação:

Curvas de altura

Floresta Ombrófila Mista

Identidade de modelos

Index terms:

Height curves

Araucaria Forest

Models identity

Histórico do artigo:

Recebido em 22 fev. 2013

Aprovado em 19 mar. 2015

Publicado em 30 set. 2015

doi: $10.4336 / 2015 . p f b .35 .83 .493$
Resumo - Este trabalho teve como objetivo testar a hipótese de que o comprimento de copa afeta a relação hipsométrica. Os dados utilizados são provenientes da medição de diâmetro a 1,30 m do solo (DAP) e respectivas alturas totais e do fuste de 337 indivíduos de Araucaria angustifolia (Bertol.) O. Kuntze de um fragmento de 15,24 ha de Floresta Ombrófila Mista situado na região urbana de Curitiba. Estas árvores foram agrupadas em classes de comprimento de copa. Foram ajustados seis modelos hipsométricos aos dados de cada classe e para todo o conjunto de dados. O melhor modelo foi escolhido com base no erro padrão da estimativa em porcentagem, Índice de Schlaegel e análise gráfica de resíduos. $\mathrm{O}$ ordenamento indicou que o modelo de melhor ajuste aos dados foi o de Trorey. O teste de identidade de modelos de Graybill aplicado ao modelo de Trorey indicou que o comprimento de copa influenciou a relação hipsométrica das araucárias da área de estudo.

\section{Efect of crown length on the hypsometric relationship of Araucaria angustifolia}

\begin{abstract}
This study aimed to test the hypothesis that crown length affects the hypsometric relationship. We used data from diameter measured at $1.3 \mathrm{~m}$ above ground level (DBH) and total heights of 337 trees of Araucaria angustifolia (Bertol.) O. Kuntze from an urban remnant of Araucaria Forest with 15.24 ha, located in Curitiba, PR, Brazil. These trees were grouped into classes of crown length. Six hypsometric models were adjusted to the data for each class and for the entire data set. The best model was chosen based on the standard error of estimate in percentage, index of Schlaegel, and graphical analysis of residuals. The ranking indicated Trorey as the best model. The models identity test of Graybill applied to the Trorey model indicated that crown length influenced the hypsometric relationship of the araucarias in the study area.
\end{abstract}


Araucaria angustifolia é uma espécie arbórea de grande porte, nativa do Brasil e que possui ampla área de distribuição, tendo maior ocorrência no sul do país onde é conhecida como pinheiro-do-Paraná. Com o começo da exploração de sua madeira considerada de altíssima qualidade no final do século XIX e o aumento das áreas de pecuária e agricultura, a área de ocorrência natural da araucária foi reduzida drasticamente, sendo encontrada atualmente em áreas fragmentadas de Floresta Ombrófila Mista em sua forma secundária, proveniente da regeneração natural ocorrida após a exploração da floresta primária (Zanon et al., 2009).

No campo florestal uma das atividades de maior importância é o inventário, que segundo Péllico Netto \& Brena (1997) visa obter informações qualitativas e quantitativas dos recursos florestais existentes em uma área, sendo assim a base para o planejamento do uso desses recursos. A determinação ou estimativa do volume total de madeira em função do diâmetro e altura das árvores é uma das atividades mais comuns no inventário florestal.

Medindo-se na parcela somente parte das alturas e todos os diâmetros, pode-se estabelecer uma relação que possibilita a estimativa da altura das demais árvores contidas na parcela, sem que haja perda de precisão (Scolforo, 2006). Vários são os fatores que influenciam a relação hipsométrica, tais como idade, sítio, tamanho da copa, densidade e espécie (Barros et al., 2002; Bartoszeck et al., 2004).

A estratificação em classes e posterior comparação dos ajustes entre as mesmas é um recurso aplicado com sucesso em vários estudos de fatores que influenciam a relação hipsométrica. Bartoszeck et al. (2004) usando tal metodologia constataram a influência dos fatores sítio e idade em plantios de bracatinga.

O conhecimento da relação hipsométrica e dos fatores que a influenciam tem grande significado prático no sistema de coleta de informações. Não existem estudos que relacionem o efeito do comprimento de copa nessa relação. Assim, este trabalho teve como objetivo estudar a influência do comprimento de copa na relação hipsométrica da araucária em seu ambiente de ocorrência natural.

\section{Caracterização da área de estudo}

A área de estudo consiste em um fragmento de 15,24 ha, com predomínio de Floresta Ombrófila Mista secundária em estágio avançado, atualmente conhecido como "Capão da Engenharia Florestal", situado no
Campus III Jardim Botânico da Universidade Federal do Paraná, em Curitiba, a aproximadamente $900 \mathrm{~m}$ de altitude. O clima da região é subtropical úmido mesotérmico com média do mês mais quente inferior a $22{ }^{\circ} \mathrm{C}$ e do mês mais frio inferior a $18{ }^{\circ} \mathrm{C}$, sem estação seca, com chuvas bem distribuídas ao longo do ano, verão brando, geadas severas e frequentes. A temperatura e a precipitação média anuais são de aproximadamente $17{ }^{\circ} \mathrm{C}$ e $1.500 \mathrm{~mm}$, respectivamente, caracterizando-se assim como clima Cfb na classificação de Köppen.

\section{Coleta de dados}

Os dados utilizados são oriundos de um censo realizado na área de estudo nos anos de 2006 e 2007. Para a realização do mesmo, a área foi dividida em blocos de $50 \mathrm{~m}$ x $50 \mathrm{~m}$, onde foram medidas, identificadas, marcadas e georreferenciadas todas as árvores com diâmetro a 1,30 $\mathrm{m}$ do solo (DAP) acima de $10 \mathrm{~cm}$.

Foram medidas as circunferências a 1,30 $\mathrm{m}$ do solo (CAP), as alturas totais e de fuste de 337 indivíduos. As CAP's foram medidas com fita métrica; as alturas totais e de fuste foram medidas com o hipsômetro Vertex III. O comprimento de copa foi obtido pela diferença entre a altura total e de fuste, sendo este considerado até o ponto onde se localizam os galhos vivos.

\section{Estratificação}

Os pares de diâmetros e alturas das 337 araucárias da área foram separados em classes de comprimento de copa, segundo o método de Sturges:

$$
n_{c}=1+3,3 * \log n
$$

Em que $n_{c}$ representa o número de classes, $n$ representa o número de observações e log é o logaritmo decimal.

O intervalo de classe é obtido pela divisão da amplitude total pelo número de classes $\left(n_{c}\right)$.

\section{Modelos testados}

Foram testados seis modelos matemáticos para cada uma das classes de comprimento de copa conforme mostra (Tabela 1), ajustados por meio do método dos mínimos quadrados. Foram utilizados modelos tradicionais encontrados na literatura florestal, utilizados por vários pesquisadores (Schneider, 1998; Bartoszeck et al., 2004).

\section{Critérios de ajuste e seleção dos modelos}

Foram utilizadas as estatísticas erro padrão da estimativa em porcentagem $\left(\mathrm{S}_{\mathrm{xy} \%}\right)$ e o índice de Schlaegel (I.A.) para escolha da equação mais precisa e posterior análise gráfica dos resíduos. As expressões dos critérios 
são:

$$
\begin{aligned}
& S_{x y}=\sqrt{Q M_{\text {res }}} \\
& S_{x y \%}=\frac{S_{x y}}{\bar{Y}} * 100 \\
& F C M=e^{0,5 *(S y x)^{2}} \\
& S_{y x_{\text {reallulado }}}=\sqrt{\frac{\sum\left(y_{i}-\hat{y}_{i}\right)^{2}}{n-p}} \\
& S_{y x \%_{\text {recalculado }}}=\frac{S_{y x_{\text {recalculado }}} * 100}{\bar{y}} \\
& I . A .=1-\left(\frac{n-1}{n-p} * \frac{S Q_{\text {residuo }}}{S Q_{\text {total }}}\right)
\end{aligned}
$$

Tabela 1. Modelos testados para a estimativa da altura total em função do DAP das araucárias.

\begin{tabular}{lll}
\hline $\mathbf{N}^{\circ}$ & \multicolumn{1}{c}{ Modelo } & \multicolumn{1}{c}{ Autor } \\
\hline 1 & $h_{i}=\beta_{0}+\beta_{1} * \frac{1}{d_{i}}+\varepsilon_{i}$ & Curtis \\
2 & $h_{i}=\beta_{0}+\beta_{1} * \frac{1}{d_{i}}+\varepsilon_{i}$ & Curtis adaptada \\
3 & $h_{i}=\beta_{0}+\beta_{1} * d_{i}+\beta_{2} * d_{i}^{2}+\varepsilon_{i}$ & Trorey \\
4 & $\ln h_{i}=\beta_{0}+\beta_{1} * \ln d_{i}+\varepsilon_{i}$ & Stoffels \& Van Soest \\
5 & $h_{i}=\left(d_{i} / \beta_{0}+\beta_{1} * d_{i}\right)^{2}+\varepsilon_{i}$ & Näslund \\
6 & $h_{i}=d_{i}^{2} /\left(\beta_{0}+\beta_{1} * d_{i}+\beta_{2} * d_{i}^{2}\right)+\varepsilon_{i}$ & Prodan \\
\hline
\end{tabular}

$\overline{\mathrm{h}_{\mathrm{i}}=\text { altura total da árvore; } \beta_{0}, \beta_{1} \text { e } \beta_{2}=\text { coeficientes do modelo; } \mathrm{d}_{1}=\text { diâmetro }}$ a $1,30 \mathrm{~m}$ do solo.

Em que: $\mathrm{S}_{\mathrm{xy}}$ é o erro padrão da estimativa; $\mathrm{S}_{\mathrm{xy} \%}$ é o erro padrão da estimativa em porcentagem; $\bar{Y}$ é a média aritmética da variável dependente; $\mathrm{QM}_{\text {res }}$ é o quadrado médio do resíduo; $n$ é o número de observações; $p$ é o número de coeficientes; $\mathrm{SQ}_{\text {residuo }}$ é a soma de quadrados dos resíduos; $\mathrm{SQ}_{\text {total }}$ é a soma de quadrados totais; $\hat{\mathrm{y}}_{\mathrm{i}}$ é a altura estimada corrigida pelo fator de Meyer; $y_{i}$ é a altura observada; $n$ representa o número de observações; $p$ é o número de coeficientes e ȳ é a média aritmética das alturas.

No caso do ajuste logarítmico (Modelo 4), antes de ser recalculado o $\mathrm{S}_{\mathrm{xy} \%}$, foi corrigida a discrepância logarítmica. Para tal correção, as variáveis dependentes estimadas foram multiplicadas pelo fator de correção de Meyer (FCM).

O índice de ajuste de Schlaegel (I.A.) é uma estatística comparável ao coeficiente de determinação ajustado $\left(\mathrm{R}^{2}\right)$, que expressa o quanto relativamente à variação total da variável a ser estimada é explicado pela regressão. O I.A. consiste em uma retransformação das predições dos valores estimados para a unidade original, servindo assim como um comparador de equações de diferentes naturezas (Schneider, 1998).

Mesmo que o $\mathrm{S}_{\mathrm{xy} \%}$ e o I.A. apresentem valores aceitáveis, a realização da análise gráfica dos resíduos permite detectar a presença de tendências em superestimar ou subestimar a variável dependente, sendo assim determinante na escolha do modelo.

Quando um conjunto de dados é separado por classes, raramente o mesmo modelo se mostra mais eficiente para todos os casos, necessitando verificar qual modelo, que na média de todas as análises, tem o melhor desempenho. Para tal, procedeu-se a um ordenamento (ranking) dos seis modelos testados para as seis classes de comprimento de copa, onde foi atribuída nota 1 à equação que apresentou melhor ajuste quanto ao $\mathrm{S}_{\mathrm{xy} \%}$, nota 2 àquela que se apresentou em segundo lugar e assim sucessivamente, sendo adotado o mesmo procedimento em relação ao I.A., sendo na sequência feita a soma dessa pontuação e o modelo que alcançou a menor soma foi considerado o mais eficiente.

Realizado o ordenamento, procedeu-se também a análise gráfica de distribuição dos resíduos do modelo que recebeu a menor pontuação, sendo na ausência de tendências recomendado como o mais adequado para uso. Os resíduos em porcentagem foram obtidos por meio da expressão:

$$
e_{i_{\%}}=\left(\frac{y_{i}-\hat{y}_{i}}{y_{i}}\right) * 100
$$

\section{Teste de identidade de Graybill}

Foi aplicado o teste de identidade de modelos proposto por Graybill (1976) para a verificação de diferenças entre as equações ajustadas para as classes, indicando ou não a influência do comprimento de copa na relação hipsométrica. Para o teste de identidade de modelos 
de Graybill (1976), decidiu-se também analisar as araucárias agrupadas em 3 classes de comprimento de copa com intervalos de $1 \mathrm{~m}$.

Esse teste consiste na redução da soma dos quadrados, permitindo verificar estatisticamente pelo teste de $\mathrm{F}$, a significância da diferença entre o total das somas dos quadrados das regressões ajustadas para cada classe isoladamente e a soma do quadrado da regressão ajustada para o conjunto total dos dados. Para o cálculo da soma de quadrados da regressão do modelo reduzido faz-se o ajuste do modelo para todos os dados e para o cálculo da soma de quadrados da regressão do modelo completo, calcula-se individualmente para cada classe e depois se faz o somatório.
A Tabela 2 contém o esquema de análise de variância para o teste de identidade proposto por Graybill.

As hipóteses testadas foram:

- $\mathrm{H}_{0}=$ o modelo reduzido ajustado não apresenta diferença em relação aos modelos ajustados por classes.

- $\mathrm{H}_{1}=\mathrm{o}$ modelo reduzido ajustado apresenta diferença em relação aos modelos ajustados por classes.

As equações de todas as classes foram comparadas entre si e posteriormente com a equação geral, envolvendo os dados de todas as classes (modelo reduzido).

Tabela 2. Esquema da análise de variância relativa ao teste de identidade.

\begin{tabular}{lcccc}
\hline Fontes de Variação & G.L. & S.Q. & Q.M. & F \\
\hline Modelo Completo & $\left(\mathrm{C}^{*} \mathrm{p}\right)$ & $\mathrm{SQ}_{\text {(completo) }}$ & \\
Modelo Reduzido & $\mathrm{p}$ & $\mathrm{SQ}_{\text {(reduzido) }}$ & \\
Redução $\left(\mathrm{H}_{0}\right)$ & $(\mathrm{C}-1) \mathrm{p}$ & $\mathrm{SQ}_{\text {(completo) }}-\mathrm{SQ}_{\text {(reduzido) }}$ & $\mathrm{SQ}_{\text {(redução) }} / \mathrm{GL}_{\text {(redução) }}$ & $\mathrm{QM}_{\text {(redução) }} / \mathrm{QM}_{\text {(residuo) }}$ \\
Resíduos & $\mathrm{N}-\left(\mathrm{C}^{*} \mathrm{p}\right)$ & $\mathrm{SQ}_{\text {(total) }}-\mathrm{SQ}_{\text {(completo) }}$ & $\mathrm{SQ}_{\text {(residuo) }} / \mathrm{GL}_{\text {(residuo) }}$ & \\
\hline Total & $\mathrm{N}$ & $\mathrm{SQ}_{\text {(total) }}$ & & \\
\hline
\end{tabular}

G.L. = grau de liberdade; S.Q. = soma dos quadrados dos resíduos; Q.M. = quadrado médio; $\mathrm{F}=$ teste $\mathrm{F} ; \mathrm{C}=$ número de classes; $\mathrm{p}=$ número de parâmetros do modelo reduzido; $\mathrm{N}$ = número de observações do modelo completo.

A não rejeição da hipótese $\mathrm{H}_{0}$ permite concluir que, a uma significância $\alpha$, as equações não diferem significativamente entre si. Assim, a equação ajustada com as estimativas dos parâmetros comuns pode ser usada para representar o conjunto de dados.

\section{Resultados e discussão}

Após a aplicação da fórmula de Sturges, foram obtidas 6 classes de comprimento de copa. A estratificação, o número de árvores por classe e o intervalo de classe, estão apresentados na Figura 1.

Para se evitar classes com um número muito pequeno de observações, as árvores com comprimento de copa maior que $3 \mathrm{~m}$ foram agrupadas em uma única classe.

A distribuição das araucárias em classes de comprimento de copa na área mostrou tendência unimodal com assimetria positiva. Machado et al. (2008), ao estudarem o comportamento da relação hipsométrica das araucárias da região, obtiveram distribuição diamétrica unimodal com comportamento semelhante ao da curva normal de Gauss.

As estatísticas básicas de dispersão dos diâmetros, alturas e comprimentos de copa da população de araucárias do Capão da Engenharia Florestal são visualizados na Tabela 3 .

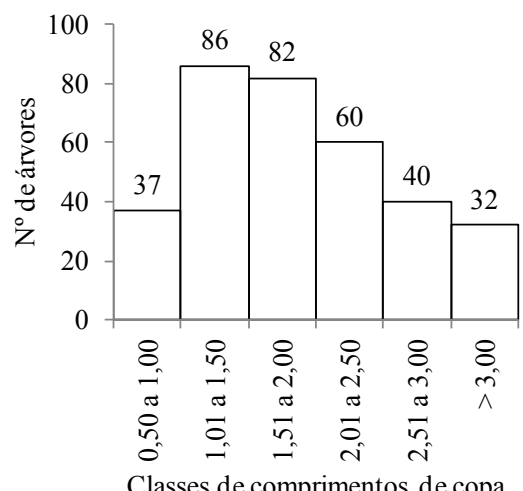

(m)

Figura 1. Distribuição da frequência das araucárias por classe de comprimento de copa. 
Observa-se na Tabela 3 que o comprimento de copa é a variável que apresenta maior dispersão em relação a média $(\mathrm{CV} \%)$ e a altura total a menor.

De acordo com Castella \& Britez (2004) a Floresta Ombrófila Mista apresenta em estágios avançados de sucessão, altura do dossel superior a 18 metros e variação diamétrica das espécies se concentrando entre $10 \mathrm{~cm}$ e $80 \mathrm{~cm}$, situação semelhante à encontrada na área do presente estudo.

\section{Avaliação dos modelos}

Na Tabela 4 são apresentados os valores do I.A. e do $\mathrm{S}_{\mathrm{xy} \%}$ obtidos pelos ajustes dos modelos em classes e pelo modelo geral.
Tabela 3. Estatísticas de dispersão dos diâmetros, alturas e comprimentos de copa das araucárias.

\begin{tabular}{lccc}
\hline Características & DAP (cm) & Ht (m) & $\begin{array}{c}\text { Comprimento de } \\
\text { copa }(\mathbf{m})\end{array}$ \\
\hline Mínimo & 26,7 & 10,9 & 0,5 \\
Máximo & 84,0 & 24,9 & 5,5 \\
Média & 53,4 & 18,1 & 1,9 \\
Desvio Padrão & 10,8 & 2,6 & 0,9 \\
CV\% & 20,3 & 14,5 & 44,5 \\
\hline
\end{tabular}

$\mathrm{Ht}=$ altura total da árvore; DAP = diâmetro a $1,30 \mathrm{~m}$ do solo; $\mathrm{CV} \%=$ coeficiente de variação.

Tabela 4. Estatísticas obtidas pelos ajustes dos modelos em classes e pelo modelo geral para as diferentes classes de comprimento de copa das araucárias.

\begin{tabular}{|c|c|c|c|c|c|c|c|c|c|c|c|}
\hline & Modelo & I.A. & $\mathbf{S}_{\mathrm{xy} \%}$ & & Modelo & I.A. & $\mathbf{S}_{\mathrm{xy} \%}$ & & Modelo & I.A. & $\mathbf{S}_{\mathrm{xy} \%}$ \\
\hline & 1 & 0,24 & 12,88 & & 1 & 0,19 & 12,89 & & 1 & 0,10 & 12,32 \\
\hline & 2 & 0,24 & 12,88 & & 2 & 0,17 & 13,01 & & 2 & 0,09 & 12,39 \\
\hline \multirow[t]{6}{*}{ Classe 1} & 3 & 0,23 & 13,00 & Classe 2 & 3 & 0,18 & 12,91 & Classe 3 & 3 & 0,12 & 12,15 \\
\hline & 4 & 0,24 & 13,03 & & 4 & 0,20 & 12,83 & & 4 & 0,10 & 12,23 \\
\hline & 5 & 0,24 & 12,91 & & 5 & 0,19 & 12,86 & & 5 & 0,10 & 12,29 \\
\hline & 6 & 0,22 & 13,02 & & 6 & 0,18 & 12,91 & & 6 & 0,11 & 12,22 \\
\hline & 1 & 0,14 & 11,69 & & 1 & 0,05 & 14,76 & & 1 & 0,23 & 10,76 \\
\hline & 2 & 0,12 & 11,85 & & 2 & 0,06 & 14,65 & & 2 & 0,25 & 10,63 \\
\hline \multirow[t]{6}{*}{ Classe 4} & 3 & 0,17 & 11,53 & Classe 5 & 3 & 0,04 & 14,81 & Classe 6 & 3 & 0,26 & 10,55 \\
\hline & 4 & 0,16 & 11,52 & & 4 & 0,04 & 14,90 & & 4 & 0,21 & 11,10 \\
\hline & 5 & 0,15 & 11,63 & & 5 & 0,05 & 14,79 & & 5 & 0,22 & 10,85 \\
\hline & 6 & 0,16 & 11,58 & & 6 & 0,07 & 14,62 & & 6 & 0,25 & 10,60 \\
\hline & 1 & 0,19 & 13,05 & & & & & & & & \\
\hline & 2 & 0,17 & 13,15 & & & & & & & & \\
\hline \multirow[t]{4}{*}{ Geral } & 3 & 0,19 & 13,03 & & & & & & & & \\
\hline & 4 & 0,19 & 13,00 & & & & & & & & \\
\hline & 5 & 0,19 & 13,03 & & & & & & & & \\
\hline & 6 & 0,19 & 13,03 & & & & & & & & \\
\hline
\end{tabular}

I.A. = índice de ajuste de Schlaegel; $\mathrm{S}_{\mathrm{xy} \%}=$ erro padrão da estimativa em porcentagem. 
É possível observar que todos os modelos testados apresentaram baixos valores do Índice de Schlaegel, o que indica uma fraca relação entre as variáveis DAP e altura total das araucárias do fragmento. Araújo et al. (2012), discutem que principalmente quando se avaliam florestas naturais, nas quais o comportamento da distribuição diamétrica é exponencial negativo - J invertido, existe uma grande concentração de indivíduos nas classes inferiores de diâmetro e uma grande variabilidade de alturas, explicando o baixo valor do coeficiente de determinação.

Os valores do $\mathrm{S}_{\mathrm{xy} \%}$ se mostraram aceitáveis, sendo pouco superiores a $10 \%$. No entanto, os índices de
Schlaegel são bastante baixos. Barros et al. (2002) analisando a relação hipsométrica em plantios de Pinus oocarpa, também encontraram coeficientes de determinação ajustados baixos para os povoamentos mais velhos e que sofreram intervenções de desbastes. No entanto, os valores do $\mathrm{S}_{\mathrm{xy} \%}$ foram sempre próximos de $10 \%$, como apresentados neste trabalho.

Após o ordenamento dos ajustes (Tabela 5), o modelo que se mostrou mais eficiente para a estimativa da altura total em função do DAP foi o de Trorey (1932), modelo 3 .

Tabela 5. Ordenamento dos ajustes dos seis modelos para as seis classes de comprimento de copa.

\begin{tabular}{|c|c|c|c|c|c|c|c|c|c|c|c|c|c|}
\hline \multirow{2}{*}{ Modelo } & \multicolumn{2}{|c|}{ Classe 1} & \multicolumn{2}{|c|}{ Classe 2} & \multicolumn{2}{|c|}{ Classe 3} & \multicolumn{2}{|c|}{ Classe 4} & \multicolumn{2}{|c|}{ Classe 5} & \multicolumn{2}{|c|}{ Classe 6} & \multirow{2}{*}{ Soma } \\
\hline & I.A. & $\mathrm{S}_{\mathrm{xy} \%}$ & I.A. & $\mathrm{S}_{\mathrm{xy} \%}$ & I.A. & $\mathrm{S}_{\mathrm{xy} \%}$ & I.A. & $\mathrm{S}_{\mathrm{xy} \%}$ & I.A. & $\mathrm{S}_{\mathrm{xy} \%}$ & I.A. & $\mathrm{S}_{\mathrm{xy} \%}$ & \\
\hline 1 & 1 & 1 & 3 & 3 & 4 & 4 & 5 & 5 & 3 & 3 & 4 & 4 & 40 \\
\hline 2 & 2 & 2 & 6 & 6 & 6 & 6 & 6 & 6 & 2 & 2 & 3 & 3 & 50 \\
\hline 3 & 5 & 5 & 4 & 4 & 1 & 1 & 1 & 1 & 5 & 5 & 1 & 1 & 34 \\
\hline 4 & 3 & 3 & 1 & 1 & 5 & 5 & 2 & 2 & 6 & 6 & 6 & 6 & 46 \\
\hline 5 & 4 & 4 & 2 & 2 & 3 & 3 & 4 & 4 & 4 & 4 & 5 & 5 & 44 \\
\hline 6 & 6 & 6 & 5 & 5 & 2 & 2 & 3 & 3 & 1 & 1 & 2 & 2 & 38 \\
\hline
\end{tabular}

I.A. = índice de ajuste de Schlaegel; $\mathrm{S}_{\mathrm{xy} \%}=$ erro padrão da estimativa em porcentagem.

Curto et al. (2014) ao estudarem a relação hipsométrica em uma floresta estacional semidecidual consideraram o modelo de Trorey como mais adequado para a estimativa das alturas totais das árvores sem estratificação.

As distribuições gráficas dos resíduos em porcentagem enquadram-se em sua maioria entre $-30 \%$ e $30 \%$, com exceção de 8 árvores que apresentaram superestimação de suas alturas, alcançando valores abaixo de $-30 \%$ de resíduo. A análise gráfica não revelou tendências dos resíduos, os quais se distribuem aleatoriamente ao longo da amplitude diamétrica (Figura 2).

Machado et al. (2008), ao ajustarem modelos hipsométricos para as araucárias dessa mesma área, não agrupando-as em classes, encontraram que o modelo de melhor performance foi o de Stoffels \& Van Soest, podendo também ser usado o modelo de Henricksen.

Na Tabela 6 estão sumarizados os coeficientes e os critérios estatísticos dos ajustes dos dados estratificados e não estratificados obtidos pelo modelo de Trorey.
Comparando-se os critérios estatísticos da regressão única com os das classes, verifica-se que apenas a classe 5 apresentou um erro maior do que quando ajustado o modelo para todas as classes em conjunto. Para as demais classes houve uma melhora nesse critério, indicando que o uso de classes é um recurso alternativo para melhorar a eficiência de modelos hipsométricos para as araucárias da região, embora essa melhora seja pequena.

A maior variação nos valores do coeficiente $\beta_{0}$, que representa onde a curva do ajuste irá interceptar o eixo-y, indicam uma maior influência do comprimento de copa nesse parâmetro do que quando analisados os coeficientes $\beta_{1}$ e $\beta_{2}$, os quais se mostram muito semelhantes, não indicando qualquer influência do comprimento de copa nesses parâmetros.

As médias das alturas e diâmetros das araucárias (Tabela 7) permitem inferir que o aumento do comprimento de copa é acompanhado pelo aumento dessas variáveis. 
Classe 1

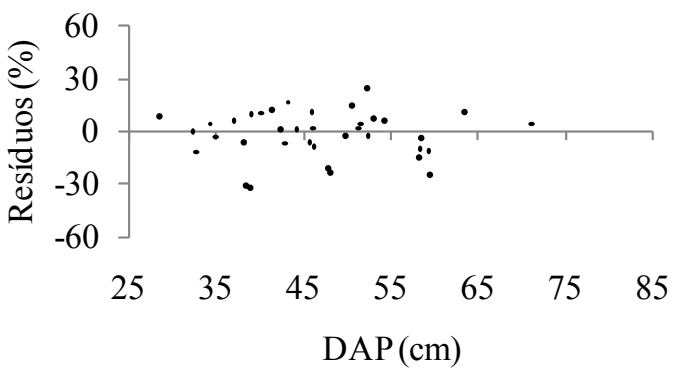

Classe 3

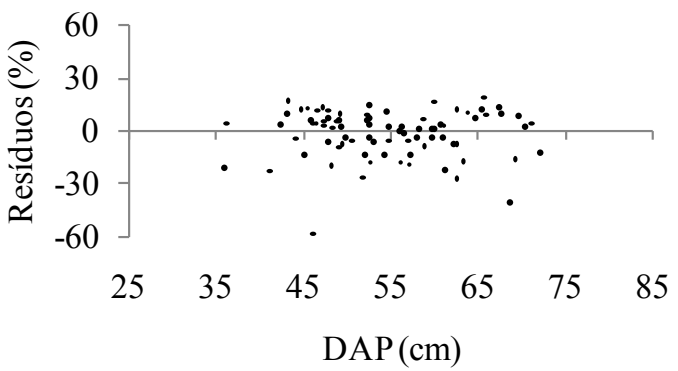

Classe 5

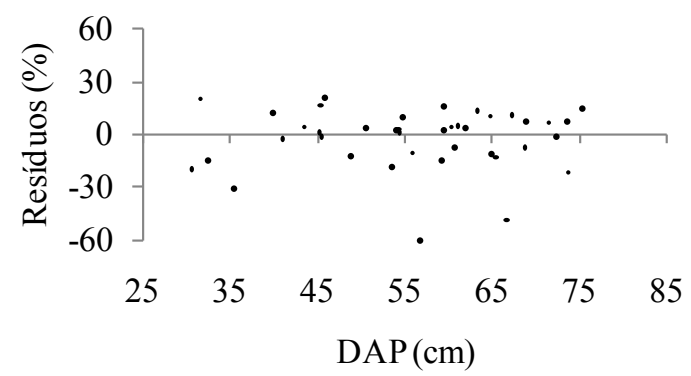

Classe 2

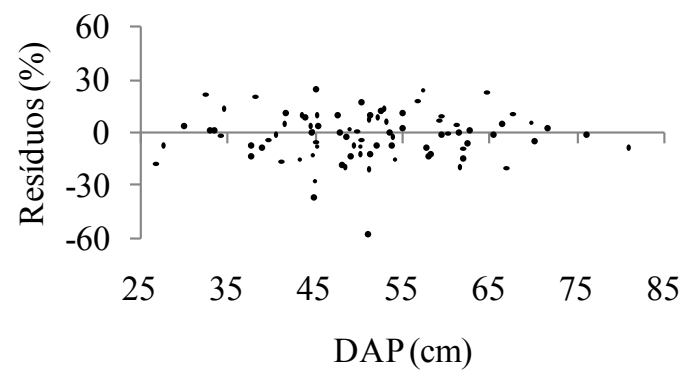

Classe 4

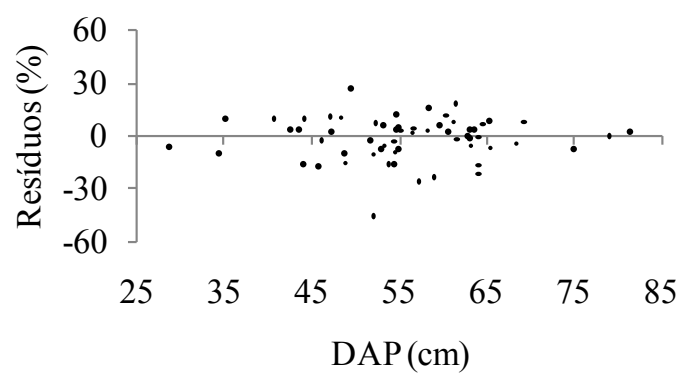

Classe 6

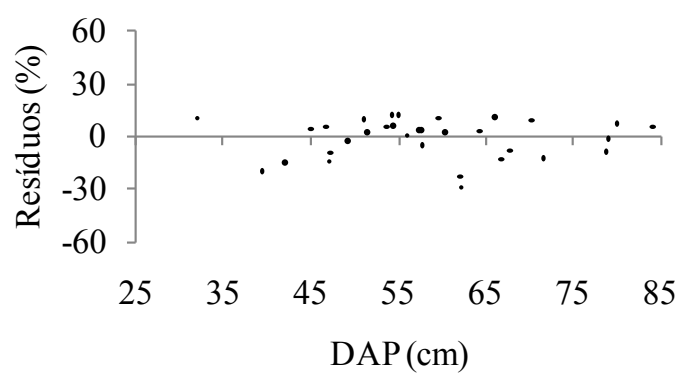

Geral

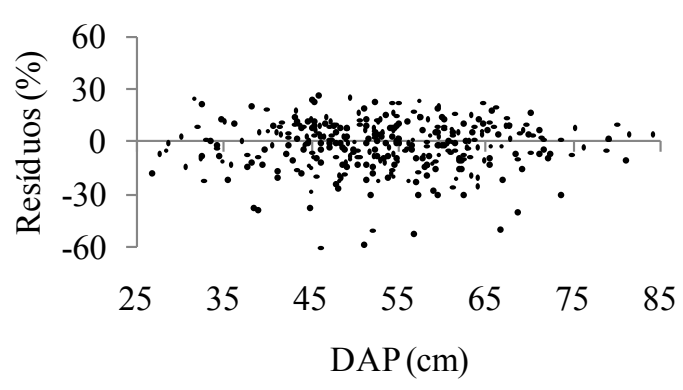

Figura 2. Distribuição dos resíduos obtidos por meio do modelo de Trorey para as seis classes de comprimento de copa e para o geral. 
Tabela 6. Coeficientes estimados e estatísticas obtidas para o modelo de Trorey para a relação hipsométrica nas diferentes classes de comprimento de copa e para o geral das araucárias.

\begin{tabular}{cccccc}
\hline Classe & $\boldsymbol{\beta}_{\mathbf{0}}$ & $\boldsymbol{\beta}_{\mathbf{1}}$ & $\boldsymbol{\beta}_{\mathbf{2}}$ & $\mathbf{S}_{\mathbf{x y} \%}$ & $\mathbf{I . A .}$ \\
\hline 1 & 1,6247 & 0,5264 & $-0,0041$ & 13,00 & 0,23 \\
2 & 10,2632 & 0,1923 & $-0,0008$ & 12,91 & 0,18 \\
3 & 23,6994 & $-0,3136$ & 0,0037 & 12,15 & 0,12 \\
4 & 15,5630 & $-0,0167$ & 0,0010 & 11,53 & 0,17 \\
5 & 5,7771 & 0,4595 & $-0,0038$ & 14,81 & 0,04 \\
6 & $-0,5248$ & 0,6447 & $-0,0046$ & 10,55 & 0,26 \\
\hline Geral & 10,2242 & 0,1925 & $-0,0008$ & 13,03 & 0,19 \\
\hline
\end{tabular}

$\beta_{0}, \beta_{1}$ e $\beta_{2}=$ coeficientes da equação; $S_{x y \%}=$ erro padrão da estimativa em porcentagem; I.A. = índice de ajuste de Schlaegel.

Tabela 7. Médias das alturas e dos diâmetros para todas as classes de comprimento de copa das araucárias.

\begin{tabular}{ccc}
\hline Classe & Ht $(\mathbf{m})$ & DAP $(\mathbf{c m})$ \\
\hline 1 & 16,7 & 46,7 \\
2 & 17,7 & 50,8 \\
3 & 18,0 & 54,5 \\
4 & 18,0 & 55,5 \\
5 & 18,9 & 55,8 \\
6 & 20,4 & 58,3 \\
\hline
\end{tabular}

$\mathrm{Ht}=$ altura total; $\mathrm{DAP}=$ diâmetro a $1,30 \mathrm{~m}$ do solo.

\section{Teste de identidade de Graybill}

O resultado do teste estatístico para a hipótese das equações das classes serem idênticas é apresentado na Tabela 8.

Tabela 8. Análise de variância das equações das 6 classes de comprimento de copa das araucárias.

\begin{tabular}{lcrll}
\hline \multicolumn{1}{c}{$\begin{array}{c}\text { Fontes de } \\
\text { Variação }\end{array}$} & G.L. & \multicolumn{1}{c}{ S.Q. } & Q.M. & F \\
\hline Modelo Completo & 18 & $111.233,6285$ & & \\
Modelo Reduzido & 3 & $111.013,0168$ & & \\
Redução $\left(\mathrm{H}_{0}\right)$ & 15 & 220,6117 & 14,7074 & $2,86^{* *}$ \\
Resíduo & 319 & 1640,3193 & 5,1421 & \\
Total & 337 & $112.873,9478$ & & \\
\hline
\end{tabular}

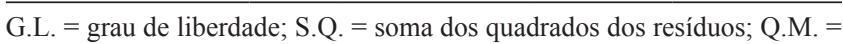
quadrado médio; $\mathrm{F}=$ teste $\mathrm{F}$.

O teste de identidade foi significativo, indicando que a estratificação em classes de comprimento de copa fez com que as equações diferissem significativamente entre si, não podendo ser utilizado o modelo reduzido com parâmetros comuns para representar a população, de forma que se deve fazer uso de uma equação diferente para cada classe de comprimento de copa.

Ao ser aplicado o teste para a comparação das equações das classes entre si, foi constatada diferença significativa apenas da equação da classe $6 \mathrm{com}$ as equações das outras classes. A classe 6 possui o maior intervalo de classe e reúne as araucárias com os maiores comprimentos de copa da população, variando entre $3 \mathrm{~m}$ e 5,5 m. O resultado do teste sugere que a estratificação realizada pelo método de Sturges resultou em classes com intervalos muito pequenos de comprimento de copa, de apenas $0,5 \mathrm{~m}$, porção pequena da árvore em relação à sua altura total.

Com objetivo de complementação dessa análise, as araucárias foram então separadas em 3 classes de comprimento de copa com intervalo de $1 \mathrm{~m}$, sendo aplicado novamente o teste de Graybill. Um número diferente de classes foi utilizado para tentar minimizar o efeito do intervalo de classe.

A Tabela 9 apresenta o resultado do teste de Graybill, quando se considerou as equações para apenas 3 classes de comprimento de copa.

Tabela 9. Análise de variância do teste de Graybill para 3 classes de comprimento de copa das araucárias.

\begin{tabular}{lcrrr}
\hline $\begin{array}{c}\text { Fontes de } \\
\text { Variação }\end{array}$ & G.L. & \multicolumn{1}{c}{ S.Q. } & Q.M. & F \\
\hline Modelo Completo & 18 & $111.165,5064$ & & \\
Modelo Reduzido & 3 & $111.013,0168$ & & \\
Redução $\left(\mathrm{H}_{0}\right)$ & 6 & 152,4896 & 25,4149 & $4,88^{* *}$ \\
Resíduo & 328 & 1708,4414 & 5,2087 & \\
Total & 337 & $112.873,9478$ & & \\
\hline
\end{tabular}

G.L. = grau de liberdade; S.Q. = soma dos quadrados dos resíduos; Q.M. $=$ quadrado médio; $\mathrm{F}=$ teste $\mathrm{F}$. 
$\mathrm{O}$ valor de $\mathrm{F}$ calculado para as 3 classes foi significativo e maior que o valor de $\mathrm{F}$ obtido para as 6 classes, indicando que a estratificação em classes de comprimento de copa com maiores intervalos fez com que as equações diferissem mais ainda entre si, induzindo que a relação hipsométrica das araucárias da região sofre influência desse fator. Ao ser aplicado o teste para a comparação das equações das classes duas a duas, a equação da classe que reúne as árvores com os maiores comprimentos de copa se diferenciou das equações das outras duas classes, sugerindo que uma estratificação em duas classes de comprimento de copa com um intervalo maior é mais adequada.

Curto et al. (2014) para comparar o ajuste geral com o ajuste realizado por espécie, parcela e classe de diâmetros para uma floresta estacional semidecidual, aplicaram o Teste $\mathrm{F}$ de Graybill e este foi não significativo a $95 \%$, indicando que as estimativas de altura total, para cada forma de ajuste, não diferiram estatisticamente daquelas obtidas sem o uso de estratificação. No entanto, verificase que a estratificação proporcionou ganhos em precisão e qualidade nos ajustes dos modelos hipsométricos. Portanto, a utilização de estratificações deve ser decidida pelo pesquisador conforme os objetivos de sua pesquisa, já que estas podem influenciar no tempo de coleta e no processamento dos dados, assim como nos custos dos mesmos.

\section{Conclusões}

O modelo de Trorey foi o mais eficiente para a estimativa da altura total de araucária em função do DAP.

Organizar os indivíduos em classes de comprimento de copa melhorou a precisão do ajuste da relação hipsométrica.

Araucárias com comprimentos de copa maiores tendem a ser mais altas e grossas.

O comprimento de copa exerceu influência na relação hipsométrica das araucárias da região.

\section{Referências}

ARAÚJO, E. J. G.; PELISSARI, A. L.; DAVID, H. C.; SCOLFORO, J. R. S.; PÉLLICO NETTO, S.; MORAIS, V. A. Relação hipsométrica para candeia (Eremanthus erythropappus) com diferentes espaçamentos de plantio em Minas Gerais, Brasil. Pesquisa Florestal Brasileira, Colombo, v. 32, n. 71, p. 257-268, 2012. DOI: 10.4336/2012.pfb.32.71.257

BARROS, D. A. de; MACHADO, S. do A.; ACERBI JUNIOR, F. W.; SCOLFORO, J. R. S. Comportamento de modelos hipsométricos tradicionais e genéricos para plantações de Pinus oocarpa em diferentes tratamentos. Boletim de Pesquisa Florestal, Colombo, n. 45, p. 3-28, jul./dez. 2002.

BARTOSZECK, A. C. P. S.; MACHADO, S. do A.; FIGUEIREDO FILHO, A.; OLIVEIRA, E. B. Dinâmica da relação hipsométrica em função da idade, do sítio e da densidade inicial de povoamentos de bracatinga da região metropolitana de Curitiba, Paraná. Revista Árvore, Viçosa, MG, v. 28, n. 4, p. 517-533, 2004.

CASTELLA, P. R.; BRITEZ, R. M. de. A floresta com araucária no Paraná: conservação e diagnóstico dos remanescentes florestais. Brasília, DF: Ministério do Meio Ambiente, 2004. 223 p.

CURTO, R. A.; LOUREIRO, G. H.; MÔRA, R.; MIRANDA, R. O. V.; NETTO, S. P.; SILVA, G. F. Relação hipsométrica em floresta estacional semidecidual. Revista de Ciências Agrárias, Belém, v. 57, n. 1, p. 57-66, jan./mar. 2014.

GRAYBILL, F. A. Theory and application of the linear model. Massachusetts: Ouxburg Press, 1976. 704 p.

MACHADO, S. A.; NASCIMENTO, R. G. M.; AUGUSTYNCZIK, A. L. D.; SILVA, L. C. R. da; FIGURA, M. A.; PEREIRA, E. M.; TÉO, S. J. Comportamento da relação hipsométrica de Araucaria angustifolia no capão da Engenharia Florestal da UFPR. Pesquisa Florestal Brasileira, Colombo, n. 56, p. 5-16, jan./jun. 2008.

PÉLLICO NETO, S.; BRENA, D. A. Inventário florestal. Curitiba, 1997. $316 \mathrm{p}$.

SCHNEIDER, P. R. Análise de regressão aplicada à Engenharia Florestal. 2. ed. Santa Maria, RS: UFSM, CEPEF, 1998.

SCOLFORO, J. R. S. Biometria Florestal: Modelos de Crescimento e Produção Florestal. Lavras, MG: UFLA/FAEPE, 2006. v. 1. 393 p.

ZANON, M. L. B.; FINGER, C. A. G.; SCHNEIDER, P. R. Proporção da dióicia e distribuição diamétrica de árvores masculinas e femininas de Araucaria angustifolia (Bert.) Kuntze., em povoamentos implantados. Ciência Florestal, Santa Maria, RS, v. 19, n.4, p. 425431, out./jun., 2009. DOI: 10.4336/2012.pfb.32.71.257 
